\title{
A prospective audit against national standards of the presentation and management of acute pancreatitis in the South of England
}

\author{
S K C Toh, S Phillips, C D Johnson
}

\begin{abstract}
Background-The incidence of acute pancreatitis shows regional variations in the UK.

Aims-To document the incidence and presentation of acute pancreatitis in hospitals in Wessex, and to audit the process and outcome of management of patients against the UK guidelines.

Methods-A prospective survey was carried out of all patients with acute pancreatitis in a one year period, in eight geographically adjacent acute hospitals in the Wessex region.

Results-186 patients with acute pancreatitis were identified, an incidence of 152 per million in the adult population. Aetiology was: gallstones $33 \%$, alcohol $20 \%$, idiopathic $32 \%$, other $15 \%$. There were 60 severe cases with 17 deaths. Age and APACHE-II score had significant relations to outcome, but delay to admission, serum amylase level, aetiology, and sex did not. The mortality rate $(9.1 \%)$ was within the audit standard of $10 \%$. Some management goals were not met: in mild cases, only one third of patients with gallstone pancreatitis had definitive treatment within four weeks. In severe cases, there was poor use of objective severity stratification (19\%), low admission rates to a high dependency unit or intensive care unit $(67 \%)$, and only $33 \%$ of patients had computed tomography. Only seven of 17 patients with severe gallstone pancreatitis had an urgent endoscopic retrograde cholangiopancreatography.
\end{abstract}

Conclusions-The incidence of clinically diagnosed acute pancreatitis in England continues to rise. Current management of acute pancreatitis is suboptimal when compared with evidence based UK guidelines but the mortality rate was within the guideline standard.

(Gut 2000;46:239-243)

Unit (816),

Southampton General

Hospital, Tremona Rd,

Southampton,

Hampshire SO16 6YD,

UK

S K C Toh

S Phillips

C D Johnson

Correspondence to:

Mr Johnson.

Accepted for publication

9 September 1999

Acute pancreatitis is an acute inflammatory condition which is fatal in $10-15 \%$ of cases. ${ }^{1-6}$ Its true incidence in the United Kingdom is difficult to determine because of regional variations: past reports have estimated an incidence of between 21 and 283 per million population. ${ }^{7-9}$ Accurate up to date audit data remain vital in evaluating the effectiveness of increasingly costly management of these patients. ${ }^{10}$ Patients with severe disease demand considerable resources in the form of radiology, endoscopy, surgery, and intensive care, with the prospect of prolonged hospital stay. ${ }^{11} 12$

The British Society for Gastroenterology (BSG), Association of Surgeons of Great Britain and Ireland, and the Pancreatic Society of Great Britain and Ireland recently commissioned a working party to produce evidence based UK guidelines for the management of acute pancreatitis. ${ }^{13}$ These allow for the first time comparison of the care received by patients against proposed audit standards, in order to identify strengths and shortcomings in diagnosis, investigation, and non-surgical treatment. The purpose of this study was to audit the current management and outcome of patients with acute pancreatitis from data collected during one year of a prospective multicentre trial in Wessex, England.

\section{Methods}

SETTING

This prospective study was conducted in the course of screening patients for a multicentre randomised controlled trial of a novel drug treatment for acute pancreatitis. ${ }^{14}$ The participating acute hospitals were Southampton General Hospital and Royal South Hants Hospital (Southampton), Royal Hampshire County Hospital (Winchester), Salisbury District Hospital, Queen Alexandra Hospital (Portsmouth), Royal Naval Hospital (Haslar), Royal Bournemouth Hospital, and Poole General Hospital. The catchment areas of the hospitals are contiguous, so the study covers a single geographical area

\section{PATIENTS}

From December 1994 to November 1995 inclusive, consecutive patients above the age of 15 admitted with acute pancreatitis into eight acute hospitals in Wessex were seen within 24 hours of admission by a single research team. The study personnel were paged by the surgical and laboratory staff of each hospital to inform them of every new patient admitted with a raised serum amylase. In addition, study personnel regularly reviewed laboratory records to identify all cases of raised serum amylase. A diagnosis of acute pancreatitis was
Abbreviations used in this paper: CT, computed tomography; ERCP, endoscopic retrograde cholangiopancreatography; ES, endoscopic sphincterotomy; HDU, high dependency unit; ITU, intensive care unit; USS, ultrasound scan. 


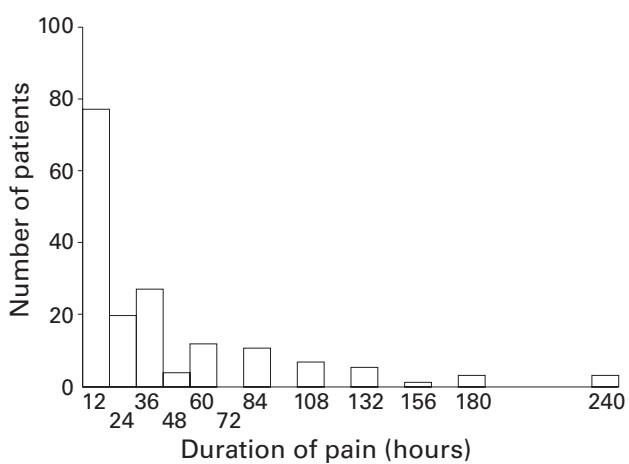

Figure 1 Time delay from onset of acute pancreatitis to admission. The values are summarised in 12 hour periods.

made if there was a clinical picture consistent with the condition and a raised serum amylase level at least three times above the upper limit of normal.

\section{CLINICAL DATA}

Data collected on admission included age, sex, delay from onset of pain to admission, and APACHE-II score. ${ }^{15}$ On discharge or death, data on severity stratification, outcome, aetiology, duration of stay in the hospital and in a high dependency unit (HDU) or intensive care unit (ITU), and imaging and endoscopy findings were obtained by careful inspection of the hospital records. A severe outcome was defined as organ failure and/or local complications according to the Atlanta criteria. ${ }^{16}$ At least one year after discharge, the records were reviewed to attempt to clarify the aetiology in the idiopathic group, and to update information on delay in management of gallstones. No steps were taken by the audit team to undertake additional clinical investigations or interventions. Surgical, gastroenterology, and radiology departments in each hospital were sent a short questionnaire to determine the provision of services listed in the UK guidelines. At least one reply was received from each hospital.

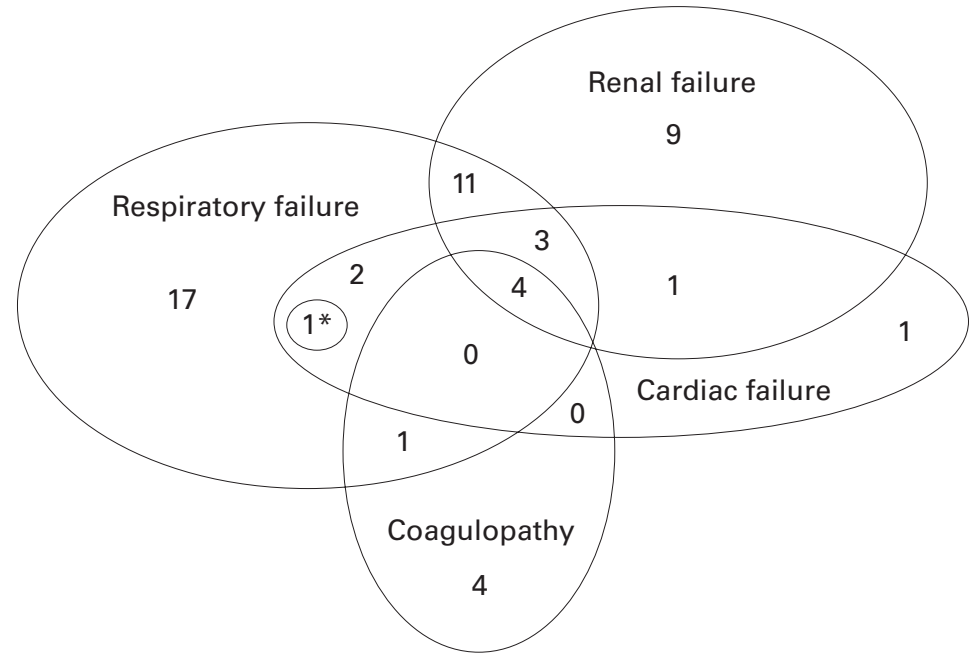

Figure 2 Systemic complications affected 54 of 186 patients with acute pancreatitis. Each line encircles the number of patients that developed the complication named within the line. Overlapping areas indicate the number that sustained two or more complications. ${ }^{\star}$ Patient with neurological, respiratory, and cardiac failure.

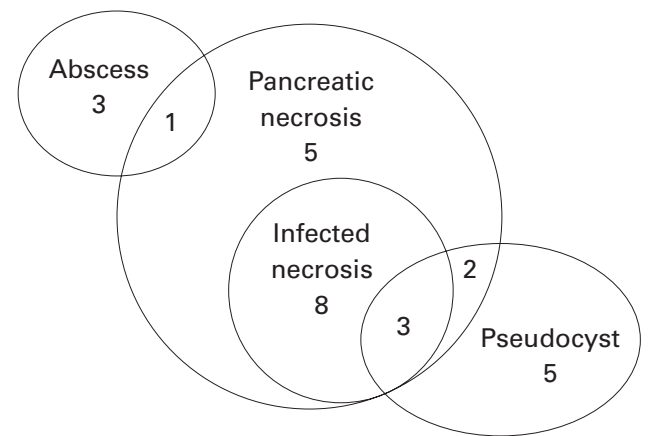

Figure 3 Local complications affected 27 of 186 patients with acute pancreatitis. Each line encircles the number of patients that developed the complication named within the line. Overlapping areas indicate the number that sustained two or more complications.

STATISTICAL METHODS

Published data $^{17}$ were used to estimate the catchment adult (over 15 years old) population of this single geographical group of hospitals. All data were collected on a proforma and SPSS for Windows 7.5 software was used for the analysis. The $t$ test was used for continuous variables and the $\chi^{2}$ test for categorical data. Significance was taken at $\mathrm{p}=0.05$.

\section{Results}

INCIDENCE OF CLINICALLY DIAGNOSED ACUTE PANCREATITIS

Over the 12 months, 186 patients with acute pancreatitis were identified. The adult population aged over 15 years served by these hospitals in 1995 was estimated to be 1222000 in 1995 giving an incidence of 152 per million population. The total population aged over 15 years in the Wessex region was 2485000 .

\section{CLINICAL DATA}

There were 80 women and 106 men. The median age was 54 years (range 15-98). The suspected aetiologies were gallstones $(n=62$; $33.3 \%)$, alcohol $(\mathrm{n}=38 ; 20.4 \%)$, drug related $(\mathrm{n}=15 ; 8.1 \%)$, endoscopic retrograde cholangiopancreatography (ERCP) $(n=5 ; 2.7 \%)$, and other $(n=6 ; 3.2 \%)$. No aetiology was found in $60(32 \%)$ patients despite an ultrasound scan (USS) and/or computed tomography (CT) in $56(93 \%)$ of these patients and drug histories taken from every patient. Fifty per cent of patients presented within 12 hours of onset, $70 \%$ within 24 hours, and 30\% after 24 hours (fig 1). There were 60 severe cases (32\%) of which 25 patients required mechanical ventilation. Seventeen of the patients with severe pancreatitis died. Figures 2 and 3 summarise the incidence of systemic and local complications. Thirty three patients had organ dysfunction only, six had local complications only, and 21 had both organ dysfunction and local complications. The median hospital stay for the severe cases was 16 days (range 2-304). Forty cases were treated in ITU or HDU, with a median stay of eight days (range 1-118). By contrast, mild cases stayed in hospital for a median of seven days (range 1-49).

The time delay from onset of symptoms to admission and the serum amylase level had no significant relation to outcome but the patient's 
Table 1 Admission data and outcome

\begin{tabular}{|c|c|c|c|c|c|}
\hline Admission data & $\begin{array}{l}\text { Outcome } \\
\text { (Atlanta) }\end{array}$ & Number & Mean & $S D$ & $p$ Value * \\
\hline \multirow[t]{2}{*}{ Age (years) } & Mild & 126 & 50.8 & 17.7 & $<0.001$ \\
\hline & Severe & 60 & 61.7 & 17.3 & \\
\hline \multirow[t]{2}{*}{ Serum amylase (IU/1) } & Mild & 126 & 2107 & 2111 & 0.065 \\
\hline & Severe & 60 & 2807 & 2508 & \\
\hline \multirow[t]{2}{*}{ APACHE II score } & Mild & 126 & 4 & 3 & $<0.001$ \\
\hline & Severe & 60 & 11 & 4 & \\
\hline \multirow{2}{*}{ Duration of pain (hours) } & Mild $†$ & 110 & 35 & 44 & 0.400 \\
\hline & Severe & 60 & 28 & 48 & \\
\hline
\end{tabular}

«Two tailed $t$ test.

†Data missing from 16 patients. population in the South of England, when compared with the incidence in Bristol in $1979^{2}$ and Nottingham in 1987-93. ${ }^{18}$ The audit against the UK guideline standards showed that several important standards of care were not met. These shortcomings could adversely affect outcome for the patients concerned.

Acute pancreatitis is a common emergency presentation, being responsible for 3\% of hospitals admissions with acute abdominal pain. ${ }^{19}$ Its incidence may be rising in the $\mathrm{UK}^{7}{ }^{70}$ but the true incidence remains unknown as these estimates are likely to be low through underdiagnosis. ${ }^{24}$ If delay in diagnosis in recorded cases can be taken as a marker of underdiagnosis, the high rate of diagnosis within 48 hours in Wessex suggests that underdiagnosis may be becoming less common as amylase estimation is now part of the routine assessment of abdominal pain. However, we have not undertaken a review of autopsy reports to look for undiagnosed fatal acute pancreatitis and we relied entirely on hyperamylasaemia in making a diagnosis, so our incidence is probably an underestimate. Bearing in mind these factors, the incidence of acute pancreatitis in the South of England appears to be higher than that recorded previously in Nottingham (128 per million, 1987-1993) ${ }^{18}$ and in Bristol (111 per million in 1979), ${ }^{2}$ but less common than in north east Scotland (242 per million). ${ }^{20}$ All these studies included cases diagnosed at autopsy whereas we report only those cases diagnosed in life. This does not affect the validity of our audit which is concerned with the management of clinically diagnosed acute pancreatitis.

In the United Kingdom, 30-50\% of cases of acute pancreatitis are related to gallstones and 15-29\% to alcohol. ${ }^{2420}$ In our audit, one third were gallstone related, and $20 \%$ were alcohol related. However, there was a higher percentage of idiopathic cases (32\%) than in Bristol $(27 \%),{ }^{2}$ north west London $(22 \%),{ }^{4}$ and north east Scotland $(20 \%){ }^{20}$ This figure is higher than the audit standard of $20-25 \% .{ }^{13}$ The guidelines state that this is not a dogmatic recommendation in the light of regional variations. However, the aetiology was recorded at discharge from hospital, and very few patients had further outpatient investigations which could have elucidated a cause in some cases.

Discussion

This study has shown an increasing incidence of acute pancreatitis in a mixed urban/rural

Table 3 Management of acute pancreatitis in Wessex compared with UK National Audit Standards ${ }^{33}$

\begin{tabular}{lll}
\hline Audit categories & Audit goals & Audit findings \\
\hline Mortality & $<10 \%(<30 \%$ in severe cases) & $9.1 \%(17)(28 \%$ of severe cases) \\
Diagnosis within 48 hours & $100 \%$ & $98.4 \% \dagger$ \\
Severity stratification within 48 hours & $100 \%$ & $19 \%(35)$ \\
Percentage of idiopathic cases & $20-25 \%$ & $32 \%(60)$ \\
Definitive treatment of gallstones in mild cases within & & $33 \%(15 / 45)$ \\
$\quad$ four weeks $\ddagger$ & $100 \%$ & $67 \%(40 / 60)$ \\
Severe cases treated in HDU or ITU & $100 \%$ & $100 \%$ \\
Radiological facilities available (USS, CT, angiography) & $100 \%$ & $33 \%(20 / 60)$ \\
Dynamic CT in severe cases days 3-10 & $100 \%$ & $41 \%(7 / 17)$ \\
Urgent ERCP in severe gallstone pancreatitis & $100 \%$ & \\
\hline
\end{tabular}

*Using an objective method of severity stratification like Glasgow criteria, Ranson criteria, APACHE II, or C reactive protein. Note that this excludes the APACHE II scoring done by the research team on every patient. †Only three cases were diagnosed after 48 hours.

$\ddagger$ Cholecystectomy and bile duct clearance or in unfit patients, endoscopic sphincterotomy only.

CT, computed tomography; ERCP, endoscopic retrograde cholangiopancreatography; HDU, high dependency unit; ITU, intensive care unit; USS, ultrasound scan. 
There was wide variation between clinicians regarding repeat ultrasonography and screening for hyperlipidaemia and other causes of pancreatitis in those without evidence of gallstones or excessive alcohol consumption. There is room for greater effort to meet the audit standard.

The use of objective scoring was well below the audit standard. The observations of this audit exclude the assessment of the APACHE-II score by the study team, which was done on each patient for the purposes of a separate clinical trial. These results were, however, available to the attending clinicians, and may have contributed to the low rate of recorded severity assessment in the case notes. We consider the low rate of recording objective assessment to be one of the most important shortcomings identified in this audit, as failure to assess severity early may result in potentially avoidable deaths. ${ }^{418}$

The management of patients with acute pancreatitis following initial resuscitation and assessment depends on their predicted severity. We found that two thirds of severe cases were treated in an ITU or HDU, as recommended by the guidelines for all severe cases. Only one third of patients had CT within 10 days, despite the availability of facilities in all hospitals. The Atlanta criteria define severe disease as any patient with a complication, including single organ failure. ${ }^{16}$ Patients with respiratory insufficiency as defined by low arterial oxygen tension early in the disease, or renal insufficiency, defined by oliguria, may respond rapidly to fluid replacement and other supportive measures, recovering without further complications. Such patients nevertheless have severe disease, because of the presence of early organ failure. Some clinicians considered a contrast enhanced CT to be unnecessary in patients with severe disease defined in this way, whose condition improved with conservative management in the first 10 days. One patient died 48 hours after admission from fulminant pancreatitis; CT would not have affected the outcome in this case. Although CT is necessary to document the extent of necrosis and fluid collections, it is expensive, not required for diagnosis in most cases, and is not used in early prediction of severity. It has not been shown to predict reliably the need for early operation. ${ }^{21}$ These arguments could be used to support a less inclusive approach to CT than that recommended in the audit standard.

Despite mounting evidence, ${ }^{22}$ some clinicians were still not fully convinced as to the efficacy of urgent endoscopic sphincterotomy (ES) in severe gallstone pancreatitis, even before the recent appearance of a (much criticised) contrary view. ${ }^{23}$ This, coupled with the lack of out of hours endoscopy in most hospitals, resulted in only seven of 17 eligible patients undergoing early ES. The small number of these patients over one year in this region suggests that provision of emergency ES is not required in every hospital, and may justify transfer of patients who require this procedure to a specialist unit where an emergency service is available.
The low rate of cholecystectomy or endoscopic bile duct clearance during the same admission shows that some patients were discharged home with their stones in place, and with the potential for recurrent pancreatitis. Unfortunately, the audit did not document how many of these patients were planned to have early definitive management of their gallstones. Nevertheless, it is worrying that definitive treatment of the gallstones was offered to only one third of patients with mild gallstone pancreatitis within four weeks of the attack, as recommended to avoid a potentially fatal second attack. A change in surgical practice will be necessary to achieve this audit standard.

The overall mortality rate was $9.2 \%$. This was within the recommended audit standard of $10 \%$ and is similar to the $8 \%$ rate reported in premortem diagnosed patients in north west

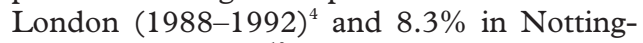
ham (1987-1993), ${ }^{19}$ although values between $3 \%{ }^{24}$ and $25 \%{ }^{25}$ have indeed been reported in the UK. A recent audit in six European countries reported a mean (SD) mortality rate of $9.2 \%(12.1 \%) .{ }^{26}$ Further afield, a retrospective study in Sowetan Africans reported a mortality of $8.1 \%{ }^{27}$ and in the United States, death rates as low as $2 \%$ have been reported. ${ }^{28}$ These variations are in part due to differences in data collection, autopsy rates, and definitions. ${ }^{4}$

In conclusion, the incidence of acute pancreatitis in England appears to be rising. The UK guidelines for the management of acute pancreatitis have set standards that allow us to assess the care of these patients. The guidelines and audit standards have helped to identify shortcomings in management. In particular, we have observed low rates of objective assessment of severity, of the use of CT in severe cases, and shortcomings in the management of mild and severe gallstone pancreatitis. The audit points to potential improvements in the management of acute pancreatitis, and illustrates the value of evidence based standard setting as a tool for measuring the quality of clinical care.

SKC Toh and S Phillips were supported by a research grant from British Biotech plc. This paper was presented as a poster at the British Society of Gastroenterology Spring Meeting 1997 and published in abstract form (Gut 1997;40(suppl 1):W42).

1 Mayer AD, McMahon MJ, Corfield AP, et al. Controlled clinical trial of peritoneal lavage for the treatment of severe acute pancreatitis. N Engl F Med 1985;312:399-404.

2 Corfield AP, Cooper MJ, Williamson RCN. Acute pancreatitis: a lethal disease of increasing incidence. Gut pancreatitis: a le $1985 ; 26: 724-9$.

3 Wilson C, McArdle CS, Carter DC, et al. Surgical treatment of acute necrotising pancreatitis. Br F Surg 1988;75:111923.

4 Mann DV, Hershman M, Hittinger R, et al. Audit of death from acute pancreatitis. Br F Surg 1994;81:890-3.

5 MRC. Death from acute pancreatitis: MRC multicentre trial of glucagon and aprotonin. Lancet 1977;ii:632-5.

6 Leese T, Holliday M, Heath DI, et al. Multicentre clinical trial of low volume fresh frozen plasma therapy in acute pancreatitis. Br F Surg 1987;74:907-11.

7 Wilson C, Imrie CW. Changing patterns of incidence and mortality from acute pancreatitis in Scotland, 1961-1985. Br F Surg 1990;77:731-4.

8 Bourke JB, Griggs J, Ebdon D. Variations in the incidence and the spatial distribution of patients with primary acute pancreatitis in Nottingham 1969-1976. Gut 1979;20:366pancre

9 Graham DF. Incidence and mortality of acute pancreatitis [letter]. BMF 1977;2:1603.

10 Carter DC. Acute pancreatitis: the value of life. Br f Surg 1993;80:1499-500.

11 Doepel M, Eriksson J, Halme L, et al. Good long-term 
results in patients surviving severe acute pancreatitis. $\mathrm{Br} \mathcal{F}$ Surg 1993;80:1583-6.

12 Fenton-Lee D, Imrie CW. Pancreatic necrosis: assessment of outcome related to quality of life and cost of management. Brf Surg 1993:80:1579-82.

13 Glazer G, Mann MV. United Kingdom guidelines for the management of acute pancreatitis. Gut 1998;42(suppl 2):S1-13.

14 Toh SKC, Johnson CD, British Pancreatitis Study Group. Lexipafant reduces mortality in a randomised placebocontrolled study in patient with severe acute pancreatitis [abstract]. Gut 1997;40(suppl 1):W46.

15 Knaus WA, Draper EA, Wagner DP, et al. APACHE II: a severity of disease classification system. Crit Care Med 1985;13:818-29.

16 Bradley EL. A clinically based classification system for acute pancreatitis. Arch Surg 1993;128:586-90.

17 de Dombal F. The acute abdomen: definitions, diseases and decisions. In: de Dombal F, ed. Diagnosis of acute abdominal pain. London: Churchill Livingston, 1991:19-30.

18 de Bolla A, Obeid M. Mortality in acute pancreatitis. Ann R Coll Surg Engl 1984;66:184-6.

19 Baneriee AK, Kaul A, Bache E, et al. Multicentre audit of death from acute pancreatitis [letter]. Br $\mathcal{F}$ Surg 1994;81: 1542 .
20 Thomson SR, Hendry WS, McFarlane GA, et al. Epidemiology and outcome of acute pancreatitis. $\mathrm{Br} \mathcal{F}$ Surg

21 Hill M, Huntington D. Computed tomography and acute pancreatitis. Gastroenterol Clin North Am 1990;19:811-42. 22 De Beaux AC, Carter DC, Palmer KR. Endoscopic retrograde cholangio-pancreatography and acute pancreatitis. Gut 1996;38:799-800.

23 Folsch UR, Nitsche R, Ludtke R, et al, German Study Group of Acute Biliary Pancreatitis. Early ERCP and papillotomy compared with conservative treatment for acute biliary pancreatitis. N Engl f Med 1997;336:237-87.

24 Cuschieri A, Wood RAB, Cumming JRG, et al. Treatment of acute pancreatitis with fresh frozen plasma. Br F Surg 1983; 70:710-12.

25 Trapnell JE, Rigby CC, Talbot CH, et al. A controlled trial of Trasylol in the treatment of acute pancreatitis. BrF Surg 1974;61:177-82.

26 Lerch M. Current trends in acute pancreatitis management (EPAP Study Group) [abstract]. Digestion 1998;59:206-7.

27 John KD, Segal I, Hassan H, et al. Acute pancreatitis in Sowetan Africans. A disease with high mortality and morbidity. Int 7 Pancreatol 1997;21:149-55.

28 Steinberg WM, Tenner S. Acute pancreatitis. $N$ Engl f Med 1994;330:1198-208. 